\title{
Evaluation of Standards and Interfering Compounds in the Determination of Phenolics by Folin-Ciocalteu Assay Method for Effective Bioprocessing of Biomass
}

\author{
Krishna Prasad Bastola1*, Yadhu Nath Guragain1, Vamsi Bhadriraju2, Praveen Venkata Vadlani1,3 \\ ${ }^{1}$ Bioprocessing and Renewable Energy Laboratory, Department of Grain Science and Industry, Kansas State University, \\ Manhattan, Kansas, USA \\ ${ }^{2}$ Department of Chemical Engineering and Material Science, University of Minnesota, Lauderdale, Minneapolis, Minnesota, USA \\ ${ }^{3}$ Department of Chemical Engineering, Kansas State University, Manhattan, Kansas, USA \\ Email: *kbastola@eosc.edu
}

How to cite this paper: Bastola, K.P., Guragain, Y.N., Bhadriraju, V. and Vadlani, P.V. (2017) Evaluation of Standards and Interfering Compounds in the Determination of Phenolics by Folin-Ciocalteu Assay Method for Effective Bioprocessing of Biomass. American Journal of Analytical Chemistry, 8, 416-431.

https://doi.org/10.4236/ajac.2017.86032

Received: May 25, 2017

Accepted: June 18, 2017

Published: June 21, 2017

Copyright $(9) 2017$ by authors and Scientific Research Publishing Inc. This work is licensed under the Creative Commons Attribution International License (CC BY 4.0).

http://creativecommons.org/licenses/by/4.0/

\section{(c) (i) Open Access}

\begin{abstract}
Folin Ciocalteu (F-C) assay is the most widely used and convenient method to determine the total phenolics content in foods, herbs, and other plant extracts. Different phenolics standards such as gallic acid, ferulic acid, chlorogenic acid, catechol, and vanillic acid have been used for calibration curves in this assay method. Comparison of these standards, in single or combination of two or more, for more accurate determination of phenolics has not been reported so far. This study tested five single phenolics and seven combinations of mixed phenolic standards to evaluate the optimal standards for F-C method. The different standards were tested to calculate the phenolic content in three known test solutions. We also evaluated interference effect of various compounds in phenolics estimation by F-C method that is usually present in the lignocellulosic biomass-derived sugar solution, and in food products along with phenolics. Finally, the optimal standards with five phenolics were used for the determination of phenolics in alkali pretreated biomass extract. The results indicated that gallic acid was the best standard among the single phenolic compounds and five phenolic compounds solution was the best standard among the mixed phenolic solutions. The presence of glucose, HMF, furfural, and vitamin-B12 did not interfere in phenolic determination; whereas ascorbic acid, tyrosine, formic acid, and acetic acid strongly interfered the results. The results also showed that biomass pelleting process did not affect the generation of phenolics in alkali pretreatment extract.
\end{abstract}

\section{Keywords}

Phenolics, Folin-Ciocalteu, Interferents, Biomass 


\section{Introduction}

Phenolic compounds are found as secondary metabolites in all plants [1] [2]. Plants use these compounds for lignin and pigment biosynthesis, protection against invading organisms (herbivores, nematodes, phytophagous insects, fungal and bacterial pathogens), growth, reproduction, and for many other important functions [3] [4]. Phenolic compounds are important constituents of human diet due to their wide range of physiological properties, such as anti-allergenic, anti-artherogenic, anti-inflammatory, anti-microbial, antioxidant, anti-thrombotic, cardioprotective, and vasodilatory effects [5]. Chemically, phenolic compounds contain an aromatic ring with one or more hydroxyl substituents, and the structural diversity ranges from simple phenolic molecules to complex polymerized compounds [6]. Lignin of lignocellulosic biomass is an example of complex phenolic polymers.

Lignocellulosic biomass is a promising feedstock for biofuels and biochemicals production because of its low cost and easy availability compared with current sugar and starchy feedstocks [7]. Basically, there are four major steps for bioconversion of lignocellulosic feedstocks to biofuels and biochemicals. The biomass is first pretreated using physical, chemical, and/or biological methods to deconstruct outer lignin layer and release carbohydrate polymers (cellulose and hemicellulose) from lignin-cellulose-hemicellose complex. The carbohydrate polymers are then enzymatically hydrolyzed to monomer sugars which are then converted to desired fuels and chemicals by aerobic and/or anaerobic fermentation. The final step is the recovery of product from the fermentation broth [8]. Each step of this biomass conversion processes is associated with several challenges; among them, the pretreatment is the central unit operation that affects the efficiency of all subsequent bioconversion processes [9]. In addition, a distinct variation in composition and structure of lignin polymer is evident among different types of biomass feedstocks [10]. This leads to the requirement of separate optimization of pretreatment process for each type of biomass [11] [12]. Fundamental understating of the fate of lignin polymer during pretreatment process is vital to develop an appropriate pretreatment method for each type of feedstock. Determination and identification of biomass lignin-derived phenolic compounds in the pretreated extract provide the key information to elucidate the mechanism of delignification during pretreatment process [10] [11]. In addition, phenolic determination is important for indirect assessment of antioxidant activities of diet [13].

Various spectroscopic and chromatographic methods are used for determining total phenolic compounds (TPC) in plant samples. Chromatographic techniques include gas chromatography (GC), gas chromatography-mass spectrometry (GC-MS), high-pressure liquid chromatography (HPLC), and liquid chromatography-mass spectrometry (LC-MS) [14]. Various spectroscopic techniques used to determine TPC are Fourier Transform Infrared (FT-IR) spectroscopy, Raman spectroscopy, and Folin-Ciocalteu (F-C) assay using UV-VIS spectroscopy [15] [16]. TPC is also indirectly measured in terms of antioxidant 
activity (AOA) of an extract as results have shown that there is a direct correlation of AOA and TPC content [17]. AOA is measured using different spectroscopic techniques such as Diphenyl-2-picryl-hydrazyl (DPPH) assay, chemiluminescence assay, Trolox equivalent antioxidant capacity, Ferric reducing-antioxidant power (FRAP) assay, and Electron spin resonance (ESPR) [18]. Chromatographic instruments are versatile tools to identify and quantify individual phenolic compounds of an extract, which are not possible by spectroscopic methods [18]. However, phenolic determination by chromatographic methods is expensive, requires considerable time for sample preparation and method development, and requires highly trained technicians. Derivatization of phenolics to volatile compounds required for GC and GC-MS analysis further makes the process more cumbersome. HPLC technique requires many standards to identify the phenolic compounds peaks in the chromatogram of test sample [19]. FT-IR and Raman Spectroscopy require chemometrics for data analysis and interpretation [20]. F-C assay using UV-VIS is the simplest and economic technique for the measurement of phenolics [21]. In F-C method, the phenolics are reacted with $\mathrm{F}-\mathrm{C}$ reagents and the reduced blue color metal complex solution is measured using UV-VIS at specified wavelength. The standard F-C procedure involved the preparation of all test solutions and reagents in water or polar organic solvents, and therefore, the method can only determine hydphilic phenolics of a sample. Kadriye et al. [22] developed a modified F-C method for the measurement of both hydrophilic and lipophilic antioxidants. In this modified method, test and standard solution were prepared in acetone, and F-C reagent diluted with isobutanol containing $0.1 \mathrm{M} \mathrm{NaOH}$ was used.

F-C assay is associated with some limitations. Many chemically similar compounds interfere with the test results. The selection of standards for calibration is critical because the reduction of F-C reagent by the standard and the phenolics in sample are comparable only if they are chemically very close [23]. Different standards such as gallic acid, ferulic acid, chlorogenic acid, vanillic acid, and catechol are being used by researchers, which generate different calibration curves and results in different numeric values of phenolics content of the same test sample [24]. Gallic acid has been widely used as an equivalent chemical in F-C method in determining total phenolics of various plant materials such as fruits, vegetables, and biomass extract [25]. Catechol, chlorogenic acid, ferulic acid, and catechin are other commonly used standards [26]. Probable reasons why most published articles used gallic acid as the most common equivalent chemical standard in FC method are: trend to follow what the literature used, high water soluble, stable in dry form, and being inexpensive. In this study, several individual phenolics and mixed phenolic standards were compared for their accuracy in determining the total phenolics content in test solutions. Therefore, a comparative evaluation of calibration curves using different standards is warranted to select the optimal standards for more accurate determination of phenolics. Luthria et al. [27] have also suggested multiple phenolic phytochemicals for the robust validation assay for accurate determination of antioxidant activity in various 
matrixes. They studied the stability and interaction effect of a five-compound phenolic mixture (caffeic acid, morin hydrate, hesperetin, catechin hydrate, and epigallocatechin gallate) assay using HPLC, and suggested that the assay can be used as a potential reference material for the accurate evaluation of TPC content and antioxidant capacity. However, to date, no work has been published on the evaluation of variable phenolic mixtures as the standards for the TPC determination by Folin Ciocalteu method.

Carlos et al. [28] compared antioxidant capacity (AOC) of mixed and individual phenolics and observed significant synergistic interaction among the mixed phenolics. Similarly, it is hypothesized that in the F-C method, the UVVIS absorption of mixed phenolics and single phenolics of same concentration would be different. The use of mixed phenolic standards instead of single phenolic standard, therefore, may result in more accurate phenolic measurement in extract containing multiple phenolic compounds.

In this study, different single phenolics and mixed phenolics (containing 2 to 11 phenolic compounds) standards were evaluated to determine the optimum calibration curves to measure the concentration of different known phenolic test solutions. These optimal calibration curves were further evaluated to measure phenolics content in a test sample containing six different phenolic compounds. The test sample was also used for the study of different interfering compounds. A wide range of compounds interfere in the determination of TPC by F-C method, and the types and level of interferents present depend on the extract natural source [29]. The selection of interfering compounds used in this study was based on the interferents present in the extract of alkali-pretreated lignocellulosic biomass, and various vitamins and amino acids present in food and feed samples along with phenolics. Some of these compounds include glucose, xylose, 5-hydroxyl methyl furfural (HMF), furfural, acetic acid, formic acid, water soluble vitamins (ascorbic acid and vitamin B12), proteins, and amino acids, in addition to phenolics from lignin degradation [10] [30]. To date, many of these compounds have not been evaluated for reactivity towards F-C reagents. Finally, the calibration curve of 5 phenolic compound standard was used to measure phenolics in alkali pretreated biomass extract.

\section{Materials and Methods}

All the chemicals used were of analytical grade and purchased either from Fisher Scientific (300 Industry Drive Pittsburgh, PA 15275) or Sigma Aldrich (3050 Spruce St. St. Louis, MO 63103). Ultra-pure water was used throughout the experiments. The name and structures of chemicals used in the experiments are depicted in Figure 1.

\subsection{Preparation of Standard and Test Solutions of Phenolics}

A series of phenolic standards solutions consisting of 1 to 11 phenolic compounds with concentrations ranging from 0.1 to $1 \mathrm{mg} / \mathrm{mL}$ were prepared. Vanillic acid, catechol, gallic acid, chlorogenic acid, and ferulic acid were selected for 

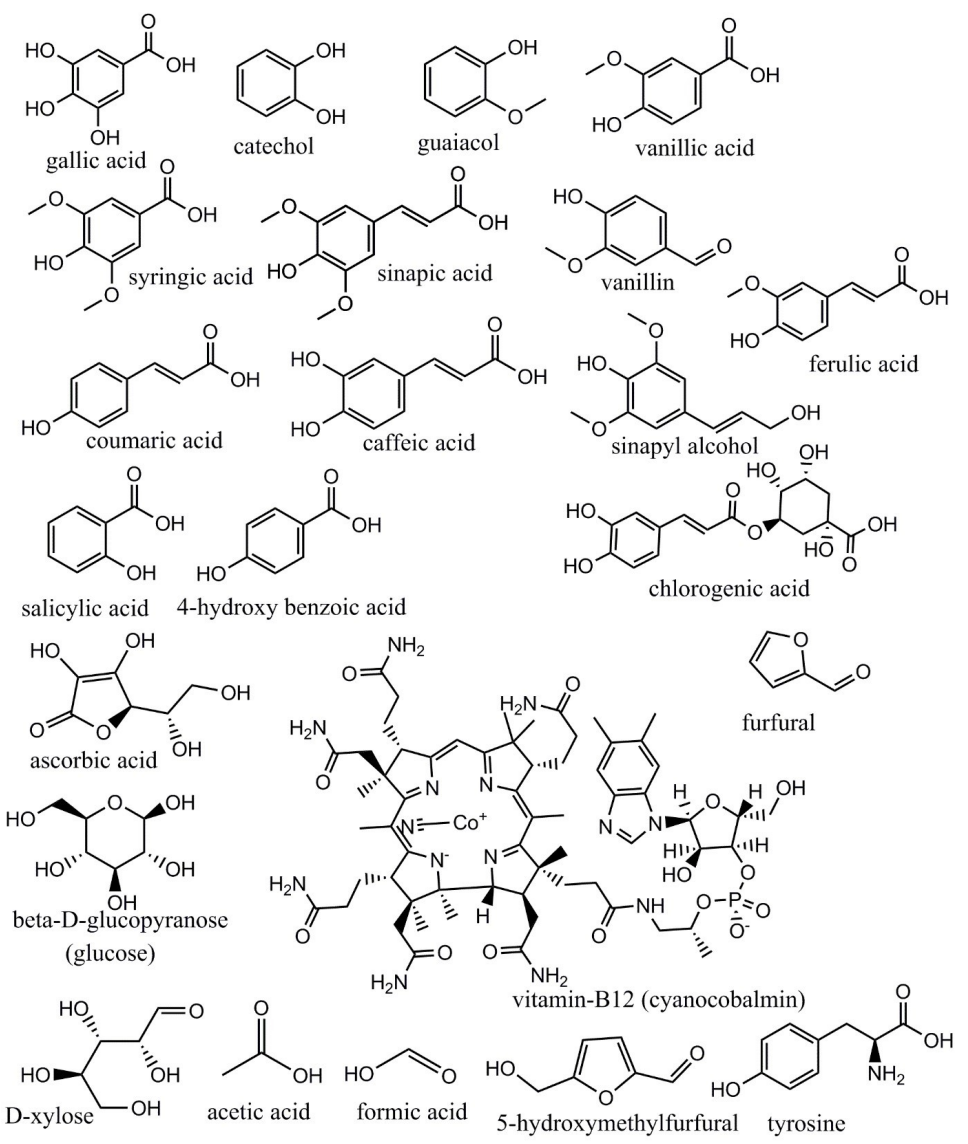

Amino acid compostion of tryptone:

alanine $2.8 \%$, arginine $3.2 \%$, asparagine $6.7 \%$, cysteine $0.3 \%$, glutamic acid $18 \%$, glycine $1.8 \%$ histidine $2.4 \%$, isoleucine $7.7 \%$, leucine $7.7 \%$, lysine $7.0 \%$, methionine $2.5 \%$, phenylalanine $4.1 \%$ proline $9.1 \%$, serine $5.1 \%$, Threonine $3.9 \%$, tryptophan $1.0 \%$, tyrosine $1.3 \%$, valine $6.0 \%$

Figure 1. Structures of phenolic compounds and interfering compounds used in the experiments

single phenolic standards. The mixed phenolic standards were prepared by mixing an equal amount of different phenolic compounds, and designated as $\mathrm{X}_{\mathrm{n}}$ where $\mathrm{n}=2,3,4,5,7,9,11$; number indicates the number of phenolic components present in the standard solution. These standards were as follow:

$\mathrm{X}_{2}=2$ phenolics (vanillic acid + catechol); $\mathrm{X}_{3}=3$ phenolics $\left(\mathrm{X}_{2}+\right.$ gallic acid); $\mathrm{X}_{4}=4$ phenolics $\left(\mathrm{X}_{3}+\right.$ guaiacol$) ; \mathrm{X}_{5}=5$ phenolics $\left(\mathrm{X}_{4}+\right.$ vanillin $) ; \mathrm{X}_{7}=7$ phenolics (gallic acid + vanillin + catechol + syringic acid + coumaric acid + sinapic acid + caffeic acid $) ; \mathrm{X}_{9}=9$ phenolics $\left(\mathrm{X}_{7}+\right.$ Sinapyl alcohol +vanillic acid $) ; \mathrm{X}_{11}=$ 11 phenolics $\left(\mathrm{X}_{9}+4\right.$-hydroxy benzoic acid + salicylic acid $)$.

\subsection{Preparation of Test Solutions of Phenolics}

A series of test solutions consisting of different phenolic compounds in equal amounts with the following compositions were prepared:

Test solution 1 (TS1) = Vanillin, vanillic acid, catechol, and guaiacol;

Test solution 2 (TS2) = Gallic acid, guaiacol, and vanillic acid;

Test solution 3 (TS3) = Catechol, gallic acid, vanillin, and guaiacol . 
Each test solution was prepared with two different total phenolics concentrations of 0.5 and $0.25 \mathrm{mg} / \mathrm{mL}$.

\subsection{Interference Testing}

The interference effect of various compounds in the measurement of phenolic solution by the F-C method was tested against a test solution containing six different phenolic compounds. A series of interfering compound solutions of the following concentration were prepared: glucose $(40 \mathrm{mg} / \mathrm{mL})$, xylose $(20 \mathrm{mg} / \mathrm{mL})$, acetic acid $(3 \mathrm{mg} / \mathrm{mL}), \mathrm{HMF}(0.5 \mathrm{mg} / \mathrm{mL})$, furfural $(0.1 \mathrm{mg} / \mathrm{mL})$, formic acid $(0.5$ $\mathrm{mg} / \mathrm{mL})$, ascorbic acid $(1 \mathrm{mg} / \mathrm{mL})$, vitamin B12 $(1 \mathrm{mg} / \mathrm{mL})$, trypton $(1 \mathrm{mg} / \mathrm{mL})$, and tyrosine $(1 \mathrm{mg} / \mathrm{mL})$. The aforementioned concentrations of glucose, xylose, acetic acid, HMF, furfural, and formic acid were selected to mimic the concentrations found in sugar solution after enzymatic hydrolysis of alkali pretreatment biomass [31]. Other interferents are usually present, along with phenolics, in various food and feed products, and their concentrations were selected arbitrarily without defined criteria.

The phenolic test solution used in the experiment consisted of gallic acid $(0.5$ $\mathrm{mg} / \mathrm{mL})$, pyrocatechol $(0.5 \mathrm{mg} / \mathrm{mL})$, 4-hydroxyl benzoic acid $(0.2 \mathrm{mg} / \mathrm{mL})$, guaiacol $(1 \mathrm{mg} / \mathrm{mL})$, syringic acid $(0.1 \mathrm{mg} / \mathrm{mL})$, and vanillin $(0.2 \mathrm{mg} / \mathrm{mL})$.

The interfering compound solutions were combined separately with equal volumes of phenolic test solutions and analyzed for the interference study.

\subsection{Determination of Phenolics by F-C Method}

The phenolics of test and standard solutions were measured spectrophotometrically according to the F-C method [32]. In brief, $0.1 \mathrm{~mL}$ of sample/standard solution was mixed well with $5 \mathrm{~mL}$ of reagent $\mathrm{A}(1: 10 \mathrm{v} / \mathrm{v}$ of $\mathrm{F}-\mathrm{C}$ reagent and deionized water) and $3.5 \mathrm{~mL}$ of reagent $\mathrm{B}(11.5 \%$, w/v aqueous sodium carbonate $)$ in a test tube, and incubated for 1 hour at $40^{\circ} \mathrm{C}$ in a water bath. The absorption spectra of the blue colored solution generated in the process was measured from 600 to $800 \mathrm{~nm}$ using Shimadzu UV-VIS 3600.

\subsection{Determination of Phenolic Content in Alkali Pretreated Biomass Extract}

Pelleted and unpelleted wheat straw, big bluestem, sorghum stalk, and corn stover biomass samples were used in the experiments. As shown in Figure 2, both pelleted and unpelleted biomass samples were alkali pretreated by mixing $10 \mathrm{~g}$ of ground biomass (size $<1 \mathrm{~mm}$ ) with $100 \mathrm{~mL}$ of $1 \%(\mathrm{w} / \mathrm{v})$ aqueous alkali, and autoclaved at $121^{\circ} \mathrm{C}$ for $30 \mathrm{~min}$, followed by filtration of biomass slurry [33]. The filtrate contained various amount of biomass lignin-derived phenolic compounds among others depending on the biomass types and processing methods. The filtrate was used to determine its phenolic contents using the $\mathrm{F}-\mathrm{C}$ reagent, and compared the phenolic content in these biomass extracts of pelleted and unpelleted samples. The solid residues were used for enzymatic hydrolysis for a separate study. 


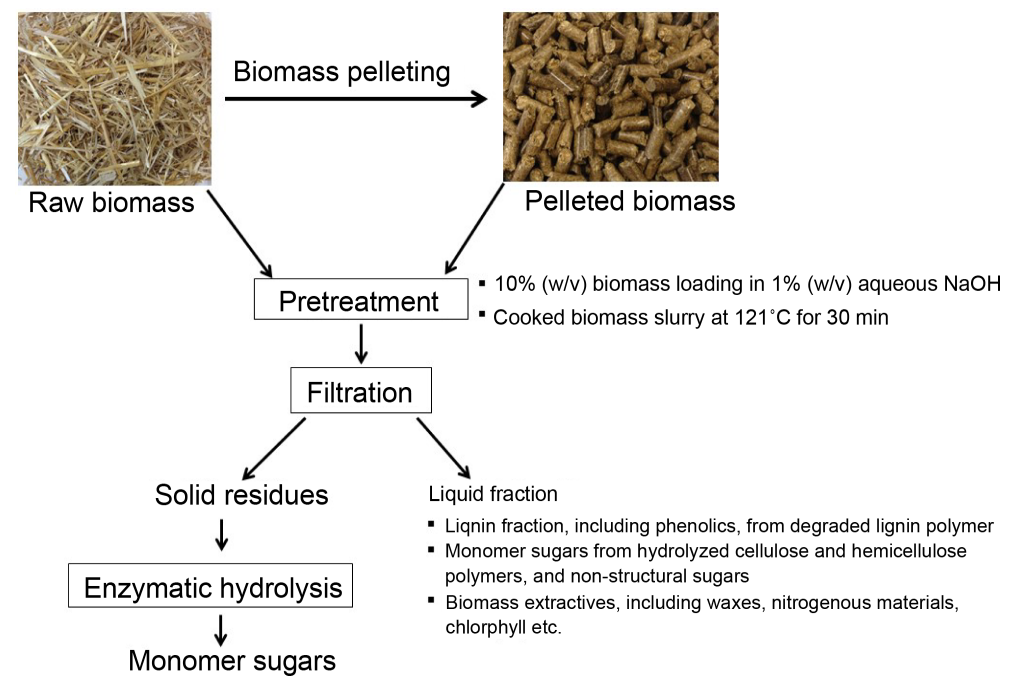

Figure 2. Schematic diagram for pelleting of lignocellulosic biomass, and subsequent alkali pretreatment and hydrolysis.

\section{Results and Discussion}

\subsection{Comparison of Single and Mixed Phenolic Standards}

Regression equations (shown in Table 1) deduced from calibration curves of the standards were used to calculate the total phenolics of known test solutions. The absolute value of difference between calculated and true concentrations of phenolics in test solutions was expressed as percentage of true concentration, and denoted as percent error. The percent errors were calculated for each standard with each test solution to determine the optimal standards from the 12 tested standards for accurate measurement of phenolics. Figure 3 revealed that five phenolic compounds standards gave the least percent error followed by gallic acid. The percent error obtained from other standards were considerably higher $(>20 \%)$; the highest deviation on the results was obtained in chlorogenic acid (single compound standard) and the mixed standards containing seven and higher phenolic compounds.

Among single phenolic standards, the calibration curve using gallic acid gave a more accurate estimation of phenolics of three test solutions compared to other single phenolic standards. The gallic acid contains three phenolic hydroxyl groups whereas other single phenolic standards (vanillic acid, chlorogenic acid, ferulic acid, and catechol) contain either one or two phenolic hydroxyl groups. A comprehensive study is needed to elucidate why the gallic acid with three phenolic hydroxyl groups is a better standard than other phenolics with one or two phenolic hydroxyl groups. In addition, the presence of other functional groups such as ether, aldehyde, $\mathrm{C}=\mathrm{C}$ bonds, carboxylic and non-phenolic hydroxyl groups may also play major roles in phenolic and F-C reagent interaction leading to the generation of different calibrations curves.

The reason to choose mixed phenolic compounds as standards is based on the hypothesis that different phenolic compounds have different interaction affinity with F-C reagent and hence different colorimetric absorption values. The stan- 
Table 1. Regression equations of 12 phenolics (single and mixed) standards obtained from their respective calibration curves.

\begin{tabular}{cc}
\hline Standards & Regression equations \\
\hline Vanillic acid & $\mathrm{Y}=1.0138 \mathrm{x}-0.0071\left(\mathrm{R}^{2}=0.999\right)$ \\
Catechol & $\mathrm{Y}=1.9037 \mathrm{x}-0.013\left(\mathrm{R}^{2}=0.9949\right)$ \\
Gallic acid & $\mathrm{Y}=1.3595 \mathrm{x}-0.003\left(\mathrm{R}^{2}=0.9946\right)$ \\
Chlorogenic acid & $\mathrm{Y}=0.66 \mathrm{x}+0.00002\left(\mathrm{R}^{2}=0.99\right)$ \\
Ferulic acid & $\mathrm{Y}=0.7564 \mathrm{x}+0.1046\left(\mathrm{R}^{2}=0.95\right)$ \\
2 phenolics & $\mathrm{Y}=1.5532 \mathrm{x}+0.0318\left(\mathrm{R}^{2}=0.99\right)$ \\
3 phenolics & $\mathrm{Y}=1.7526 \mathrm{x}+0.0421\left(\mathrm{R}^{2}=0.99\right)$ \\
4 phenolics & $\mathrm{Y}=1.8556 \mathrm{x}-0.0509\left(\mathrm{R}^{2}=0.99\right)$ \\
5 phenolics & $\mathrm{Y}=1.5237 \mathrm{x}-0.0226\left(\mathrm{R}^{2}=0.99\right)$ \\
7 phenolics & $\mathrm{y}=0.8773 \mathrm{x}-0.0625\left(\mathrm{R}^{2}=0.99\right)$ \\
9 phenolics & $\mathrm{y}=1.0022 \mathrm{x}-0.1866\left(\mathrm{R}^{2}=0.98\right)$ \\
11 phenolics & $\mathrm{y}=0.7771 \mathrm{x}-0.0751\left(\mathrm{R}^{2}=0.98\right)$ \\
\hline
\end{tabular}

2 phenolics $=$ mixed phenolic standard solutions containing 2 phenolic compounds; similary 11 phenolics $=$ mixed phenolic standard solutions containing 11 phenolic compounds.

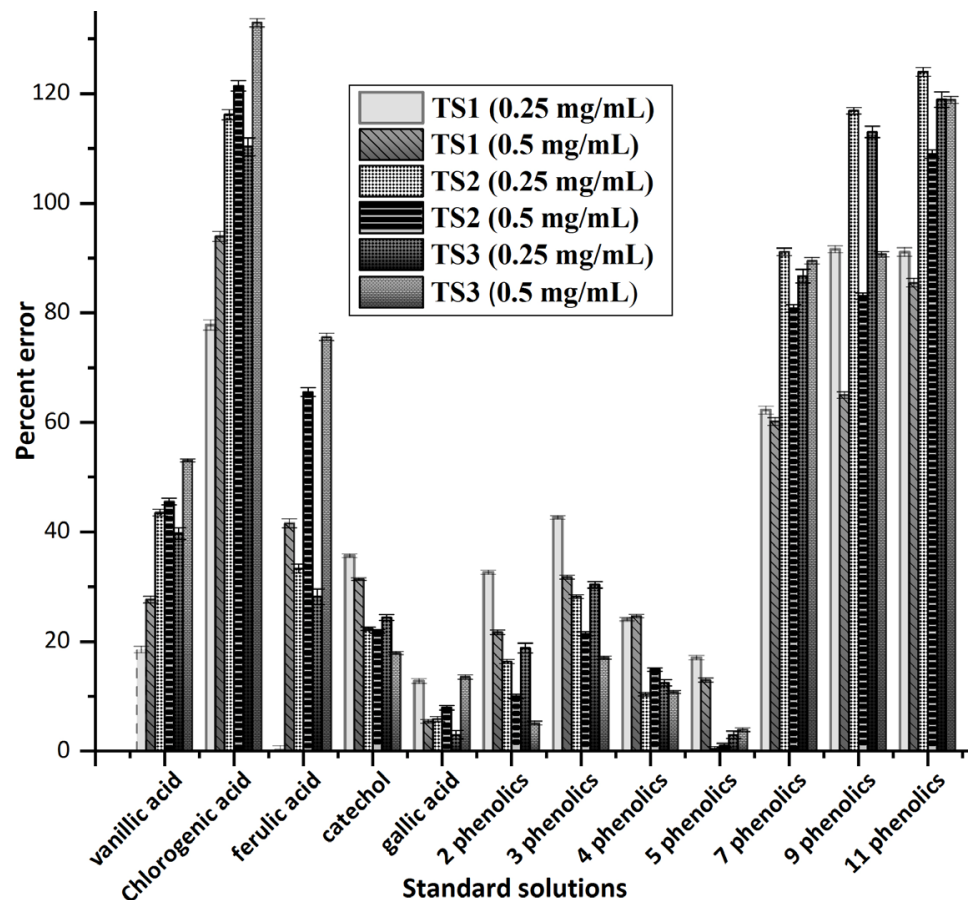

Figure 3. Comparison of percent errors of three test solutions using the regression equations of 12 (single and mixed) phenolic standards. The absolute value of difference between calculated and true concentrations of phenolics in test solutions was expressed as percentage of true concentration, and denoted as percent error. TS1 = Test solution 1 (Vanillin, vanillic acid, catechol, and guaiacol); TS2 = Test solution 2 (Gallic acid, guaiacol, and vanillic acid); and TS3 = Test solution 3 (Catechol, gallic acid, vanillin, and guaiacol). All the phenolic compounds in the test solutions were present in equal amounts. Each test solution was prepared with two concentrations $(0.25$ and $0.5 \mathrm{mg} / \mathrm{mL})$. Data are average values of triplicate experiments, and error bars represent test solutions standard deviation. The percent error of test solutions were obtainced based on the true concentration of test solutions and their concentration values calculated using regression equations of different standard solutions. 
dard containing many phenolic compounds with different absorption values is expected to give the overall absorption values close to the test solutions containing multiple phenolic compounds. It is not understood why the standard solution containing five phenolic compounds gave lowest percent errors for the test solutions when compared with the other aforementioned mixed phenolic standards. More research with different combinations of five phenolic compounds is needed to revalidate that five phenolic compounds is optimal among the mixed phenolic standards. It was observed that standards with seven and higher phenolic compounds gave very high percent error than the standards containing lower number of phenolic compounds. This could be due to the interactions among the phenolic compounds leading to either negative synergism or additive effects on electron donation to metal complex of FC reagent, and producing lower or higher absorption signal [34].

\subsection{Study of Different Interfering Compounds in Phenolic Measurement by F-C Method}

Compounds such as sugars (glucose, xylose), furan compounds (Furfural, HMF), amino acids (tyrosine, leucine), peptone (trypton), organic acids (acetic acid, formic aid), and vitamins (ascorbic acid, vitamin B-12) were separately mixed with a phenolic test solution containing six different phenolic compounds and their interference effect was determined. The phenolic content of the test solution was also determined using the aforementioned regression equations for further revalidation of optimal standards, and was found that only standards of gallic acid and five phenolic compounds calculated concentrations close to the true concentration of the test solution with percent error of 9.8 and $9.2 \%$, respectively (data not shown). This further illustrated that gallic acid and five phenolic compounds were better standards for phenolic determination when compared to other individual and mixed phenolic standards.

The UV absorptions of phenolic test solutions with and without the addition of interfering compounds measured from 600 to $800 \mathrm{~nm}$ wavelength are shown in Figure 4. The results showed that phenolic solutions containing glucose, furfural or HMF have no significant difference in absorption intensities compared to the control (test solution with only phenolics), indicating that these compounds are not reducing agent, and hence did not interfere in the determination of phenolic compounds. Maximum absorption peak of phenolic solution with xylose, formic acid, and acetic acid, were 7\%, 9\%, and 13\% less, respectively, than control solution. Out of two sugars (glucose and xylose) used in this study, only xylose significantly interfered the adsorption despite the xylose concentration $(10 \mathrm{mg} / \mathrm{ml})$ was half that of the glucose $(20 \mathrm{mg} / \mathrm{mL})$. Further study is needed to investigate why xylose (pentose sugar) is behaving as a stronger reducing agent than glucose (hexose sugar). Out of two organic acids, acetic acid concentration as acetate ion in the phenolic solution was three times more than formate ion, but the reduction in absorbance by acetic acid was only 1.4 times more than formic acid. This indicated that formic acid being a strong reducing agent strongly interacts with the F-C reagents compared to acetic acid which is a 


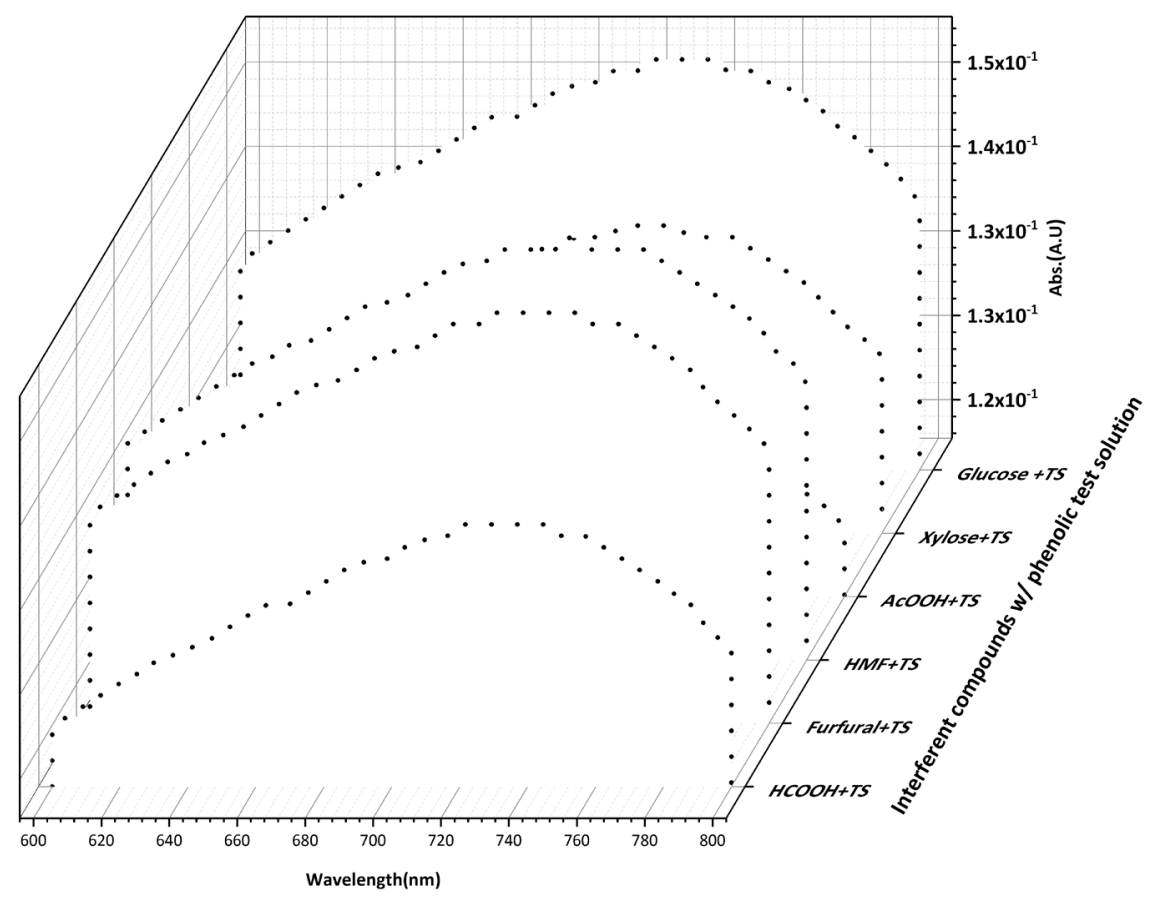

Figure 4. Measurement of absorbance of phenolic test solution mixed with equal volume of individual interfering compounds. Phenolic test solution (TS) = solution containing 7 different phenolic compounds. The concentration of Acetic acid, formic acid, xylose, and glucose in the test solutions were $1.5,0.025,10,20 \mathrm{mg} / \mathrm{mL}$, respectively. The concentration of the test solution was $1.25 \mathrm{mg} / \mathrm{mL}$. All the UV-VIS spectra were taken after 10 times dilution of the test solution. The absorbance of Test solution (TS) alone and the test solution with glucose interferent compound had the same values and hence to make the graph celarly readable, the TS data was not included in the graph. The plot of glucose with test solution is also considered as the plot for the test solution alone.

weak reducing agent. Carbon dioxide is formed during the redox reaction of formate ion and $\mathrm{F}-\mathrm{C}$ reagent as shown in the following equation [35].

$$
\begin{aligned}
& \mathrm{HCOOH}^{-}+\mathrm{M}^{n+} \rightarrow \mathrm{CO}_{2}+\mathrm{H}^{+}+\mathrm{M}^{(n-2)+}, \\
& E^{0}=-0.46 \mathrm{~V}(\mathrm{pH}=8)(\mathrm{M} \text { is a metal ion })
\end{aligned}
$$

Figure 5 shows that the maximum absorptions of phenolic solution with tyrosine and ascorbic acid were $21 \%$ and $33 \%$ more than control solution (test solution only with phenolics), whereas no significant difference in absorptions was observed for tryptone and vitamin-B12 compared to control. It was also found that leucine and isoleucine did not interfere the phenolic determination (data not shown).

It was reported that two amino acids, tyrosine and tryptophan, strongly interfered the phenolic determination [36]. Tryptone was studied to assess the interference effects of protein and peptides [37]; tryptone is a peptone from enzymatic digest of casein, composed of 18 types of amino acids as shown in Figure 1. Our results also confirmed the interference effect of tyrosine. Tryptone con tains both tyrosine (1.3\%) and tryptophan (1\%); however, no significant interference effect in phenolic determination was observed, which might be due to the low percentage composition of these interfering amino acids, indicating that a 


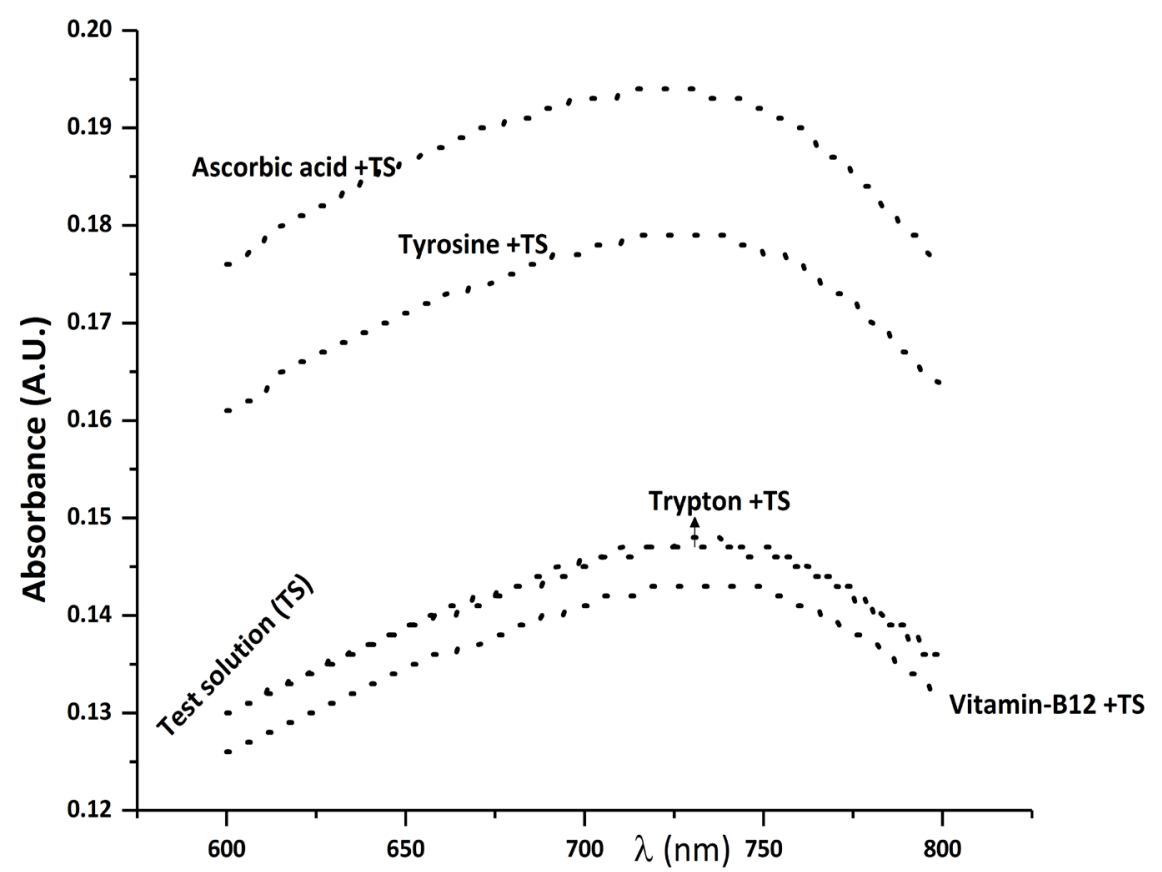

Figure 5. Measurement of absorbance of phenolic test solution mixed with equal volume of individual interfering compounds. Phenolic test solution (TS) = solution containing 7 different phenolic compounds. The concentration of vitamin B-12, tryptone, tyrosine, and ascorbic acid in the test solutions were $0.5 \mathrm{mg} / \mathrm{mL}$, and the concentration of the test solution was $1.25 \mathrm{mg} / \mathrm{mL}$. All the UV-VIS spectra were taken after 10 times dilution of the test solution.

protein or peptide interferes phenolics determination only when they contain high percent of tyrosine and tryptophan. The strong interfering effects of these two amino acids are probably due to the presence of highly ionizable hydroxyl (in tyrosine) and imino group (tryptophan), which strongly interact with the F-C reagent.

Out of two vitamins evaluated in this study (vitamin B-12 and ascorbic acid), vitamin B-12 (molecular formula: $\mathrm{C}_{63} \mathrm{H}_{88} \mathrm{CoN}_{14} \mathrm{O}_{14} \mathrm{P}$ ) is a complex organometallic compound consisting of numerous functional groups such as amide, hydrox$\mathrm{yl}$, and heterocyclic aromatic ring. Ascorbic acid is a ketolactone containing two ionizable hydroxyl groups. Vitamin B-12, despite containing numerous functional groups and aromatic rings, is not interfering compound as they do not contain any ionizable functional groups whereas ascorbic containing two ionizable hydroxyl group is a strong interfering compound.

The UV-VIS absorption spectra (Figure 6) of F-C reagent combined individually with ascorbic acid, vitamin B12, trypton, and tyrosine without any phenolic compounds further indicated that ascorbic acid and tyrosine strongly reacted with the reagents, whereas insignificant interaction was observed with vitamin-B12 and trypton. This observation also validates the above results that ascorbic acid and tyrosine are strong interference compounds, but not vitamin-B12 and trypton.

The study further indicated that there are two types of interference com- 


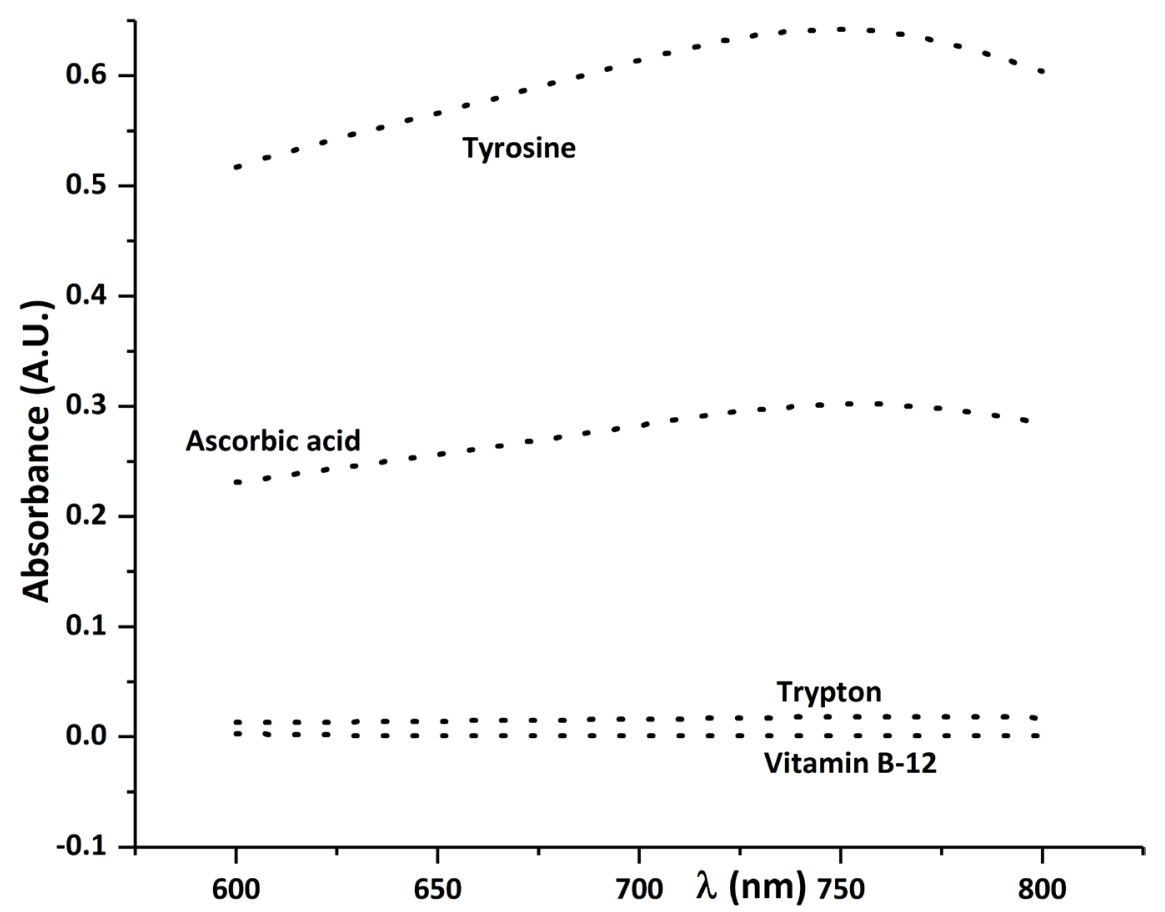

Figure 6. UV-VIS absorption spectra of interferents treated with F-C reagent. The concentration of each interferents was $0.5 \mathrm{mg} / \mathrm{mL}$. All the UV-VIS spectra were taken without further dilution of the test solution.

pounds. The compounds, such as organic acids, decrease the absorption intensity of phenolic compounds by interfering with the electron donating capacity of the phenolics. The second type of compounds, such as ascorbic acid and tyrosine, react with the F-C reagent and absorb UV-VIS radiation in the same wavelength regions of phenolic analytes and give misleading enhanced absorption of analyte.

\subsection{Phenolic Content in Alkali Pretreated Biomass Extract}

In our previous study, we evaluated and compared the feedstock quality of pelleted and unpelleted biomass for bioethanol production and found that pelleting process improve the pretreatment and hydrolysis efficiency to release sugars from different biomass samples, including corn stover, big bluestem, wheat straw, and sorghum stalk [31]. In this study, the effect of biomass pelleting on the phenolic content of alkali pretreated biomass extract was evaluated. It was hypothesized that the alkali pretreated pelleted biomass extract will have higher phenolics than unpelleted biomass extract. The basis for the hypothesis was that the pelleting process partially deconstructed the biomass leading to better delignification during alkali pretreatment in pelleted biomass compared with unpelleted biomass. The aforementioned regression equation of five phenolic compounds (Table 1) was used to calculate the concentration of phenolic compounds in each sample. Figure 7 shows that phenolic compounds present in the biomass extract were statistically equal at $95 \%$ confidence level in pelleted and unpelleted samples for all biomass types. However, the phenolic content among 
different types of biomass were significantly different from each other. Pretreatment extract of corn stover and sorghum stalks had relatively higher phenolics content than wheat straw and big bluestem. This results indicated that pelleting process did not affect the generation of phenolics during alkali pretreatment of biomass, which was in contrary to our hypothesis.

\section{Conclusion}

Our study indicated that selection of suitable standard leads to more accurate estimation of phenolics. Mixed phenolic standards are another option for the optimal determination of phenolics. Gallic acid was found to be the best standard among the single phenolic standards used in this study. Among the mixed standards, five phenolics standards consisting of vanillic acid, catechol, gallic acid, guaiacol, and vanillin in equal amounts estimated phenolics more accurately than other tested mixed phenolic standards. The interference study indicated that glucose, HMF, furfural, and vitamin B-12 did not interfere in phenolic estimation whereas ascorbic acid, tyrosine, formic acid, and acetic acid strongly interfered. A commonly acceptable optimal standard establishment is warranted for total phenolics analysis, so that results can be compared rationally by all the researchers. The optimal standards with five phenolics used for the determination of phenolics in alkali pretreated biomass extract indicated that biomass pelleting did not affect the generation of phenolics during alkali pretreatment.

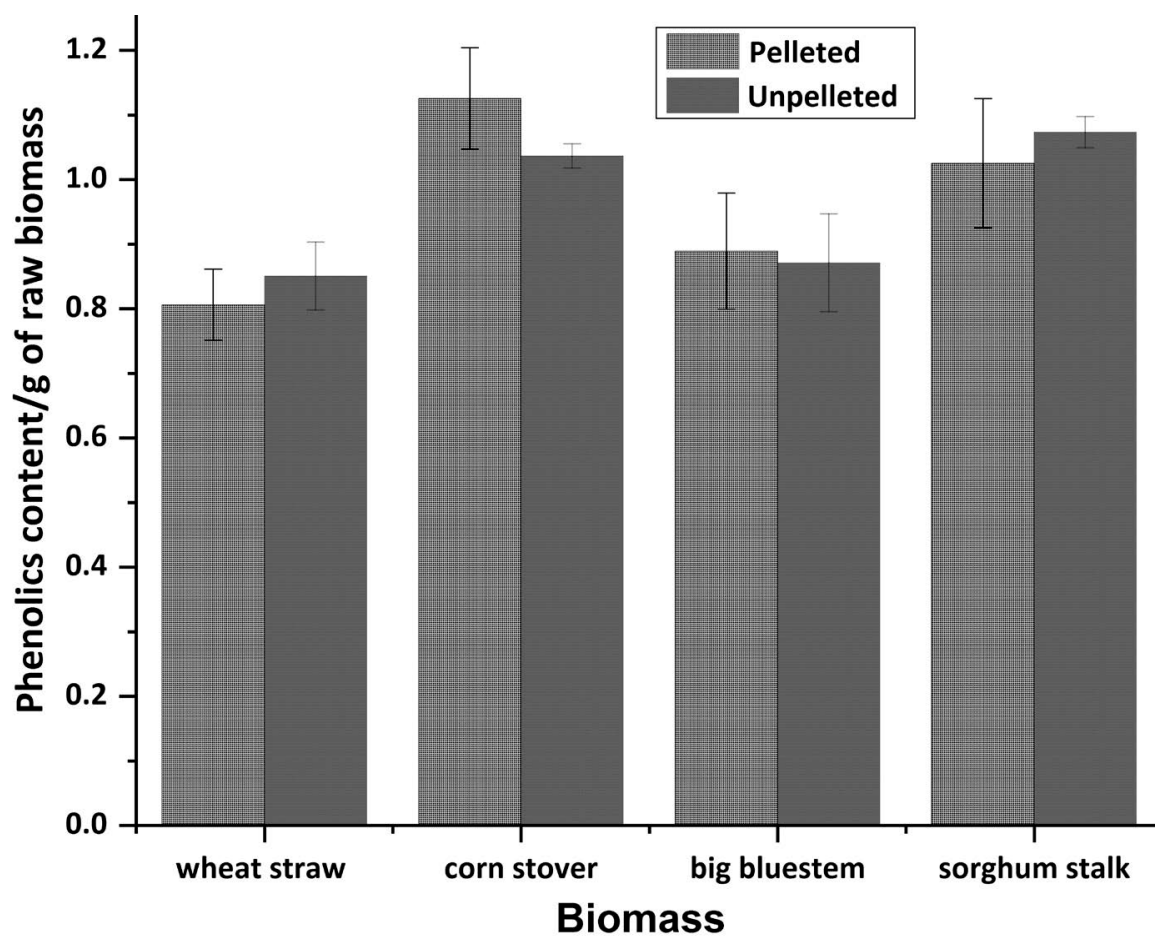

Figure 7. Comparison of phenolic contents of alkali pretreated extract of pelleted and unpelleted biomass samples. The biomass extract was the filtrate of pretreated biomass slurry. Biomass pretreatment was carried out by mixing $10 \mathrm{~g}$ ground biomass (size $<1$ $\mathrm{mm}$ ) and $100 \mathrm{~mL}$ of $1 \%(\mathrm{w} / \mathrm{v})$ sodium hydroxide solution in $500 \mathrm{ML}$ Erlenmeyer flask followed by autoclaving at $121^{\circ} \mathrm{C}$ for 30 minutes. 


\section{Acknowledgements}

This work was funded by Development Initiative Competitive Grands Program (BRDI; grant number: 2012-10008-20263). Author P. V. V. thanks the Lortscher Endowment for their financial support. The article is contribution No. 16-202-J from Kansas Agriculture Experimental Station (KAES).

\section{References}

[1] Antolovich, M., Prenzler, P., Robards, K. and Ryan, D. (2000) Sample Preparation in the Determination of Phenolic Compounds in Fruits. Analyst, 125, 989-1009. https://doi.org/10.1039/b000080i

[2] Macheix, J. and Fleuriet, A. (1990) Fruit Phenolics. CRC Press, Boca Raton.

[3] Bhattacharya, A., Sood, P. and Citovsky, V. (2010) The Roles of Plant Phenolics in Defence and Communication during Agrobacterium and Rhizobium Infection. Molecular Plant Pathology, 11, 705-719. https://doi.org/10.1111/j.1364-3703.2010.00625.x

[4] Lattanzio, V., Lattanzio, V.M. and Cardinali, A. (2006) Role of Phenolics in the Resistance Mechanisms of Plants against Fungal Pathogens and Insects. Phytochemistry: Advances in Research, 661, 23-67.

[5] Balasundram, N., Sundram, K. and Samman, S. (2006) Phenolic Compounds in Plants and Agri-Industrial By-Products: Antioxidant Activity, Occurrence, and Potential Uses. Food Chemistry, 99, 191-203. https://doi.org/10.1016/j.foodchem.2005.07.042

[6] Bravo, L. (1998) Polyphenols Chemistry, Dietary Sources, Metabolism, and Nutritional Significance. Nutrition Reviews, 56, 317-333. https://doi.org/10.1111/j.1753-4887.1998.tb01670.x

[7] Guragain, Y.N., Probst, K.V. and Vadlani, P.V. (2016) Fuel Alcohol Production. In: Wrigley, C., Corke, H., Seetharaman, K. and Faubion, J., Eds., Encyclopedia of Food Grains, 2nd Edition, Academic Press, Oxford, 235-244. https://doi.org/10.1016/B978-0-12-394437-5.00137-6

[8] Guragain, Y.N., De Connick, J., Husson, F., Durand, A. and Rakshit, S.K. (2011) Comparison of Some New Pretreatment Methods for Second Generation Bioethanol Production from Wheat Straw and Water Hyacinth. Bioresource Technology, 102, 4416-4424. https://doi.org/10.1016/j.biortech.2010.11.125

[9] Guragain, Y.N. and Vadlani, P.V. (2016) Importance of Biomass-Specific Pretreatment Methods for Effective and Sustainable Utilization of Renewable Resources. Biotechnology and Biochemical Engineering, Springer Nature, Scientific Publishing Services, 207-215

[10] Guragain, Y.N., Herrera, A.I., Vadlani, P.V. and Prakash, O. (2015) Lignins of Bioenergy Crops: A Review. Natural Product Communications, 10, 201-208.

[11] Guragain, Y.N., Wang, D. and Vadlani, P.V. (2016) Appropriate Biorefining Strategies for Multiple Feedstocks: Critical Evaluation for Pretreatment Methods, and Hydrolysis with High Solid Loading. Renewable Energy, 96, 832-842. https://doi.org/10.1016/j.renene.2016.04.099

[12] Guragain, Y.N., Bastola, K.P., Madl, R.L. and Vadlani, P.V. (2016) Novel Biomass Pretreatment Using Alkaline Organic Solvents: A Green Approach for Biomass Fractionation and 2,3-Butanediol Production. BioEnergy Research, 9, 643-655. https://doi.org/10.1007/s12155-015-9706-y

[13] Jacobo-Velázquez, D.A. and Cisneros-Zevallos, L. (2009) Correlations of Antioxi- 
dant Activity against Phenolic Content Revisited: A New Approach in Data Analysis for Food and Medicinal Plants. Journal of Food Science, 74, R107-R113. https://doi.org/10.1111/j.1750-3841.2009.01352.x

[14] Carrasco-Pancorbo, A., Cerretani, L., Bendini, A., Segura-Carretero, A., GallinaToschi, T. and Fernández-Gutiérrez, A. (2005) Analytical Determination of Polyphenols in Olive Oils. Journal of Separation Science, 28, 837-858. https://doi.org/10.1002/jssc.200500032

[15] Schulz, H. and Baranska, M. (2007) Identification and Quantification of Valuable Plant Substances by IR and Raman Spectroscopy. Vibrational Spectroscopy, 43, 1325. https://doi.org/10.1016/j.vibspec.2006.06.001

[16] Kumar, T. and Jain, V. (2015) Appraisal of Total Phenol, Flavonoid Contents and Antioxidant Potential of Folkloric Lannea coromandelica Using in Vitro and in Vivo Assays. Scientifica, 2015, Article ID: 203679. https://doi.org/10.1155/2015/203679

[17] Alam, M.N., Bristi, N.J. and Rafiquzzaman, M. (2013) Review on in Vivo and in Vitro Methods Evaluation of Antioxidant Activity. Saudi Pharmaceutical Journal, 21, 143-152. https://doi.org/10.1016/j.jsps.2012.05.002

[18] Robbins, R.J. (2003) Phenolic Acids in Foods: An Overview of Analytical Methodology. Journal of Agricultural and Food Chemistry, 51, 2866-2887. https://doi.org/10.1021/jf026182t

[19] Šeruga, M., Novak, I. and Jakobek, L. (2011) Determination of Polyphenols Content and Antioxidant Activity of Some Red Wines by Differential Pulse Voltammetry, HPLC and Spectrophotometric Methods. Food Chemistry, 124, 1208-1216. https://doi.org/10.1016/j.foodchem.2010.07.047

[20] Cozzolino, D. (2015) Infrared Spectroscopy as a Versatile Analytical Tool for the Quantitative Determination of Antioxidants in Agricultural Products. Foods and Plants Antioxidants, 4, 482-497. https://doi.org/10.3390/antiox4030482

[21] Agbor, G.A., Vinson, J.A. and Donnelly, P.E. (2014) Folin-Ciocalteau Reagent for Polyphenolic Assay. International Journal of Food Science, Nutrition and Dietetics, 2326-3350.

[22] Berker, K.I., Ozdemir-Olgun, F.A., Ozyurt, D., Demirata, B. and Apak, R. (2013) Modified Folin-Ciocalteu Antioxidant Capacity Assay for Measuring Lipophilic Antioxidants. Journal of Agricultural and Food Chemistry, 61, 4783-4791. https://doi.org/10.1021/jf400249k

[23] Appel, H.M., Govenor, H.L., D’ascenzo, M., Siska, E. and Schultz, J.C. (2001) Limitations of Folin Assays of Foliar Phenolics in Ecological Studies. Journal of Chemical Ecology, 27, 761-778. https://doi.org/10.1023/A:1010306103643

[24] Prior, R.L., Wu, X. and Schaich, K. (2005) Standardized Methods for the Determination of Antioxidant Capacity and Phenolics in Foods and Dietary Supplements. Journal of Agricultural and Food Chemistry, 53, 4290-4302. https://doi.org/10.1021/jf0502698

[25] Proteggente, A.R., Pannala, A.S., Paganga, G., Buren, L.V., Wagner, E., Wiseman, S. Put-Fvd Dacombe, C. and Rice-Evans, C.A. (2002) The Antioxidant Activity of Regularly Consumed Fruit and Vegetables Reflects Their Phenolic and Vitamin C Composition. Free Radical Research, 36, 217-233.

https://doi.org/10.1080/10715760290006484

[26] Everson, C., Miller, G. and Quackenbush, F. (1957) Comparison of Antioxidants for Fats on an Equivalent Molar Basis. Journal of the American Oil Chemists Society, 34, 81-83. https://doi.org/10.1007/BF02638026 
[27] Luthria, D.L. and Vinyard, B.T. (2008) Development of a Potential Reference Material for Evaluating Antioxidant Activity. Journal of AOAC International, 91, 506510.

[28] Palafox-Carlos, H., Gil-Chávez, J., Sotelo-Mundo, R.R., Namiesnik, J., Gorinstein, S. and González-Aguilar, G.A. (2012) Antioxidant Interactions between Major Phenolic Compounds Found in Ataulfo'mango Pulp: Chlorogenic, Gallic, Protocatechuic and Vanillic Acids. Molecules, 17, 12657-12664. https://doi.org/10.3390/molecules171112657

[29] Everette, J.D., Bryant, Q.M., Green, A.M., Abbey, Y.A., Wangila, G.W. and Walker, R.B. (2010) Thorough Study of Reactivity of Various Compound Classes toward the Folin-Ciocalteu Reagent. Journal of Agricultural and Food Chemistry, 58, 81398144. https://doi.org/10.1021/jf1005935

[30] Hannus, K. and Pensar, G. (1973) Silvichemicals in Technical Toilage. I. Water Steam Distilled Oil from Pine Material. Paperi ja Puu (Paper and Timber), 55, 509519.

[31] Guragain, Y.N. (2015) Sustainable Bioprocessing of Various Biomass Feedstocks: 2,3-Butanediol Production Using Novel Pretreatment and Fermentation. Ph.D. Dissertation, Kansas State University, Manhattan.

[32] Hendrik, P. and Yvonne de Jong-Van, B. (2011) Chemical Determination of Phenolic Compounds.

http://prometheuswiki.publish.csiro.au/tiki-index.php?page=Chemical+determinati on+of+phenolic+compounds

[33] Guragain, Y.N., Wilson, J., Staggenborg, S., McKinney, L., Wang, D. and Vadlani, P.V. (2013) Evaluation of Pelleting as a Pre-Processing Step for Effective Biomass Deconstruction and Fermentation. Biochemical Engineering Journal, 77, 198-207. https://doi.org/10.1016/j.bej.2013.05.014

[34] Pinelo, M., Manzocco, L., Nuñez, M.J. and Nicoli, M.C. (2004) Interaction among Phenols in Food Fortification: Negative Synergism on Antioxidant Capacity. Journal of Agricultural and Food Chemistry, 52, 1177-1180. https://doi.org/10.1021/jf0350515

[35] Mota, C.S., Rivas, M.G., Brondino, C.D., Moura, I., Moura, J.J., González, P.J. and Cerqueira, N.M. (2011) The Mechanism of Formate Oxidation by Metal-Dependent Formate Dehydrogenases. Journal of Biological Inorganic Chemistry, 16, 1255 1268. https://doi.org/10.1007/s00775-011-0813-8

[36] Sánchez-Rangel, J.C., Benavides, J., Heredia, J.B., Cisneros-Zevallos, L. and JacoboVelázquez, D.A. (2013) The Folin-Ciocalteu Assay Revisited: Improvement of Its Specificity for Total Phenolic Content Determination. Analytical Methods, 5, 5990 5999. https://doi.org/10.1039/c3ay41125g

[37] Anonymous Amino Acid Composition of Tryptone. Technical Information from US Biological Life Sciences. http://www.usbio.net/item/T8750 
Submit or recommend next manuscript to SCIRP and we will provide best service for you:

Accepting pre-submission inquiries through Email, Facebook, LinkedIn, Twitter, etc. A wide selection of journals (inclusive of 9 subjects, more than 200 journals)

Providing 24-hour high-quality service

User-friendly online submission system

Fair and swift peer-review system

Efficient typesetting and proofreading procedure

Display of the result of downloads and visits, as well as the number of cited articles Maximum dissemination of your research work

Submit your manuscript at: http://papersubmission.scirp.org/

Or contact ajac@scirp.org 